\title{
Affine / Photometric Invariants for Planar Intensity Patterns
}

\author{
Luc Van Gool ${ }^{1}$, Theo Moons ${ }^{1 \star}$ and Dorin Ungureanu ${ }^{1}$ \\ Katholieke Universiteit Leuven, ESAT - MI2, \\ K. Mercierlaan 94, B-3001 Heverlee, BELGIUM
}

\begin{abstract}
The paper contributes to the viewpoint invariant recognition of planar patterns, especially labels and signs under affine deformations. By their nature, the information of such 'eye-catchers' is not contained in the outline or frame - they often are affinely equivalent like parallelograms and ellipses - but in the intensity content within. Moment invariants are well suited for their recognition. They need a closed bounding contour, but this is comparatively easy to provide for the simple shapes considered. On the other hand, they characterize the intensity patterns without the need for error prone feature extraction. This paper uses moments as the basic features, but extends the literature in two respects: (1) deliberate mixes of different types of moments to keep the order of the moments (and hence also the sensitivity to noise) low and yet have a sufficiently large number to safeguard discriminant power; and (2) invariance with respect to photometric changes is incorporated in order to find the simplest moment invariants that can cope with changing lighting conditions which can hardly be avoided when changing viewpoint. The paper gives complete classifications of such affine / photometric moment invariants. Experiments are described that illustrate the use of some of them.
\end{abstract}

\section{Introduction}

A lot of research has been put into the extraction of invariants for planar shapes under geometrical deformations [5, 6]. Most work has focused on the shapes' contours. For certain applications, however, it would be more effective if one could use invariants derived from the intensity patterns bounded by the contour. For example, if one is to recognize labels or traffic signs, the contours will typically contain little information. If affine distortions are considered, many of them will be affinely equivalent (e.g. all parallelograms or all ellipses). Contour invariants will be difficult to apply to many of the patterns and therefore moment invariants are considered. Affine distortions are the most general type of geometric transformations that can be considered in this case, because expressions of moments that are projectively invariant do not exist [12]. Note the natural complementarity: with complex outlines contour invariants can be used and it would be

* Postdoctoral Research Fellow of the Belgian National Fund for Scientific Research (N.F.W.O.). 
difficult to extract closed contours as required by the moments; in the case of non-discriminant outlines of the forementioned, simple parametric types closed contours can be fitted rather easily for the calculation of the moments, whereas contour invariants are of little use.

The goal being invariant characterisation of the intensity patterns, care has to be taken of photometric changes as well. When the camera changes its position relative to the pattern, its intensities will in general change. This paper contains a complete and systematic classification of invariants for the combined effect of affine deformations and photometric changes. A second concern is to keep the order of the moments low, since high orders introduce more noise. To that effect, the mixing of different kinds of moments and the combination of coplanar patterns are considered. Different kinds of moments have been defined (geometric, Legendre, Zernike, rotational, etc.). The reported work is based on the traditional "geometric" moments and only moments up to the second order are considered. These choices are in keeping with the results of noise sensitivity tests [11].

Of course, the paper adds to a large body of literature on moments and moment invariants. It is impossible to give a complete overview here (for a partial review, see Prokop and Reeves [8]). Nevertheless, there are a number of contributions which are directly related to the presented work. Maitra [4] and Abo-Zaid et al. [1] discussed variations of Hu's metric and scaling moment invariants [3] that are also invariant under global scaling of the intensity. Another strand of research has concentrated on deriving moment invariants under affine transformations $[2,9,10]$. Reiss [9] combined affine and photometric invariance and his work comes closest to that reported. Nevertheless, most of the moment invariants that are given here are novel.

The paper is organized as follows: first, notation and terminology is established in Section 2. In Section 3 the affine/photometric invariants are systematically classified according to the highest order of the moments involved. For each case, invariants under affine transformations, photometric invariants, as well as combined affine/photometric invariants are given. Section 4 then reports on ongoing experiments with these moment invariants. Section 5 summarizes the results.

\section{Affine / Photometric Transformations}

\subsection{Definitions and Notations}

If $x$ and $y$ stand for the row and column coordinates of an image with intensities $i(x, y)$ and a planar object's closed contour $C$ circumscribes the region $\Omega$ in the image, then

$$
M S_{C p q}=\iint_{\Omega} x^{p} y^{q} d x d y \quad \text { and } \quad M I_{C p q}=\iint_{\Omega} i(x, y) x^{p} y^{q} d x d y
$$

are the shape $(p, q)$-moment and intensity $(p, q)$-moment resp.. Both are said to be of order $p+q$. For brevity, in the sequel " $n$ th-order moments" stand for the 
set of moments up to and including the $n$ th-order moments. If $M_{C p q}$ rather than $M S_{C p q}$ or $M I_{C p q}$ is specified, this is to mean that the corresponding expression can be used with either of them. Notation often will be simplified to $M S_{p q}$ and $M I_{p q}$ if there is only one contour involved.

The moment invariants discussed in the paper are deliberately made function of both shape moments and intensity moments. This mix lowers the required order of the moments and thus contributes to their robustness. With " $n$ th-order moment invariants" will be meant moment invariants that combine moments up to order $n$.

\subsection{Geometric and Photometric Changes}

The geometric deformations considered are affine transformations

$$
\left(\begin{array}{l}
x^{\prime} \\
y^{\prime}
\end{array}\right)=\left(\begin{array}{ll}
a_{11} & a_{12} \\
a_{21} & a_{22}
\end{array}\right)\left(\begin{array}{l}
x \\
y
\end{array}\right)+\left(\begin{array}{l}
b_{1} \\
b_{2}
\end{array}\right)
$$

with $|A|=a_{11} a_{22}-a_{12} a_{21} \neq 0$. This implies that the camera is relatively far from the object.

Two kinds of photometric changes are considered: pure scaling

$$
I^{\prime}(x, y)=s I(x, y)
$$

and scaling combined with an offset

$$
I^{\prime}(x, y)=s I(x, y)+o
$$

The assumptions that the camera is relatively far from the object and that the object is planar can greatly simplify the analysis of the photometric changes. Typically light sources are far from the objects as well. The geometry of light reflection is the same for all points in that case, i.e. they share the same angles of light incidence and camera viewing direction. Also for the more sophisticated models of diffuse reflection the change in camera or light position will in that case result in an overall scaling of intensity [7]. The offset allows to better model the combined effect of diffuse and specular reflection [14] and has been found to give better performance [9].

The actions of the photometric and affine changes on the moments come out to commute. Hence, one might first normalize against one type of transformation and then against the other. Alternatively, one may normalize against one and switch to the use of invariants for the other. To some extent, this latter strategy typically is what has happened in the literature. The photometric offset can e.g. be eliminated through the use of intensity minus average intensity and the photometric scale parameter can be eliminated by normalizing the resulting intensity's variance [9]. After these normalisations one then has to deal with affine deformations exclusively. The resulting affine invariants may look simpler than the ones given here, but the inherent complexity is at least comparable. However, the normalisation steps are quite expensive computationally, since they 
require a pixel-wise modification. Not normalising means that one has to deal with larger numbers (original intensities and coordinates instead of deviations from average values), but this is outweighed by far by only using lower order moments.

\subsection{Geometric and Photometric Effects on Moments}

As already mentioned before, only moments up to 2 nd order will be considered. How do these transformations affect the value of the moments? The affine transformation (1) changes the vector

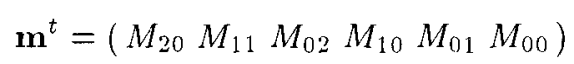

of second-order moments as

$$
\mathbf{m}^{\prime}=\mathbf{T}_{\mathrm{m}} \mathbf{m}
$$

where

$$
\mathbf{T}_{m}=|A|\left(\begin{array}{cccccc}
a_{11}^{2} & 2 a_{11} a_{12} & a_{12}^{2} & 2 a_{11} b_{1} & 2 a_{12} b_{1} & b_{1}^{2} \\
a_{11} a_{21} & a_{11} a_{22}+a_{12} a_{21} & a_{12} a_{22} & a_{11} b_{2}+a_{21} b_{1} & a_{12} b_{2}+a_{22} b_{1} & b_{1} b_{2} \\
a_{21}^{2} & 2 a_{21} a_{22} & a_{22}^{2} & 2 a_{21} b_{2} & 2 a_{22} b_{2} & b_{2}^{2} \\
0 & 0 & 0 & a_{11} & a_{12} & b_{1} \\
0 & 0 & 0 & a_{21} & a_{22} & b_{2} \\
0 & 0 & 0 & 0 & 0 & 1
\end{array}\right)
$$

with $|A|=a_{11} a_{22}-a_{12} a_{21}$ as before. As can be seen from the transformation matrix, the 0th-order moment $M_{00}$, as well as the 1 st-order moments (including $M_{00}$ ) can also be considered in isolation. Observe that shape and intensity moments transform in exactly the same way under affine transformations.

If the model of the photometric changes is restricted to pure scaling, then

$$
M I_{i j}^{\prime}=s M I_{i j} \quad \text { and } \quad M S_{i j}^{\prime}=M S_{i j}^{\prime}
$$

The shape moments are trivially invariant, because they do not involve intensities. To obtain photometric intensity moment invariants, it suffices to take the ratio of two intensity moment invariants. Shape and intensity moments can thus be used separately.

If the intensity changes by a scale factors and an offset o, then

$$
M_{I i j}^{\prime}=s M_{I i j}+o M_{S i j} \quad \text { and } \quad M_{S i j}^{\prime}=M_{S i j} .
$$

The result is a 2-dimensional group of transformations acting on the vectors $\left(M I_{i j}, M S_{i j}\right)^{T}$. Again the shape moments are trivially invariant. However, the intensity moments can no longer be used in isolation. Observe that the photometric changes act identically on all intensity moments, irrespective of their order.

The actions of the photometric and the geometric transformations commute. As a consequence, the overall group of affine/photometric transformations is a direct product of the affine group and the group of intensity transformations. This implies that combined affine/photometric invariants exist if the number 
of moments surpasses the sum of the orbit dimensions of both actions taken separately, and that the invariants are found as common expressions in the sets of affine and photometric moment invariants separately. A Lie group theoretical strategy was used to classify all independent affine/photometric moment invariants up to the second order. This classification is given in the next section. Space restrictions do not allow to discuss the generation of these invariants. The interested reader is referred to [13] for details on the classification strategy.

\section{Affine / Photometric Moment Invariants}

This section classifies the independent moment invariants up to the second order. For each order, affine, photometric (for both models), and combined affine/photometric invariants are given, which are mixtures of both shape and intensity moments. If the invariants only involve shape moments, combinations of different patterns (different bounding contours) are considered, until an invariant involving an intensity moment is found, because the assumption is that the useful information is contained in the intensity pattern. In practice, additional contours can be generated by making an invariant subdivision of the pattern under consideration. Two examples are given in Fig. 1. An elliptical pattern can be divided into the original ellipse and an ellipse with the same center of gravity, orientation and eccentricity, but having half the size along each axis. Similarly, a parallelogram shaped pattern can be subdivided into e.g. the whole pattern and the pattern inside the diamond that emerges by connecting the midpoints on each side.
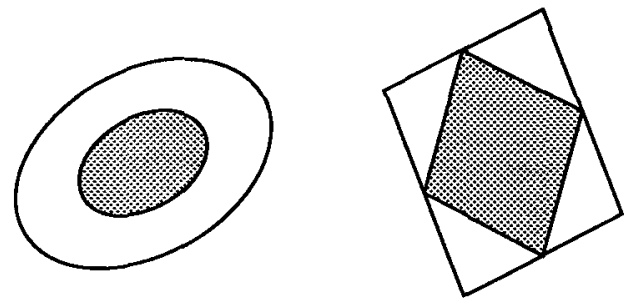

Fig. 1. Invariant subdivisions of an ellipse and a parallelogram.

\subsection{Zeroth-order Moment Invariants}

Affine Invariants : $M I_{00} / M S_{00}$

Photometric Invariants.

Photometric scaling: $M S_{C 00} ; M S_{D 00}$ and $M I_{C 00} / M I_{D 00}$ 
Photometric scaling + offset $: M S_{C 00} ; M S_{D 00} ; M S_{E 00}$, and $\frac{M I_{C 00} M S_{D O 0}-M I_{D O 0} M S_{C 00}}{M I_{C 00} M S_{E 00}-M I_{E 00} M S_{C 00}}$

\section{Affine / Photometric Invariants.}

Photometric scaling : $M I_{C 00} / M I_{D 00}$ and $M S_{C 00} / M S_{D 00}$

Photometric scaling + offset $: M S_{C 00} / M S_{D 00} ; M S_{C 00} / M S_{E 00}$, and $\frac{M I_{C 00} M S_{D 00}-M I_{D 00} M S_{C 00}}{M I_{C 00} M S_{E O 0}-M I_{E Q 0} M S_{C 00}}$

\subsection{First-order Moment Invariants}

Affine Invariants : $M I_{C 00} / M S_{C 00} ; M S_{D 00} / M S_{C 00}$

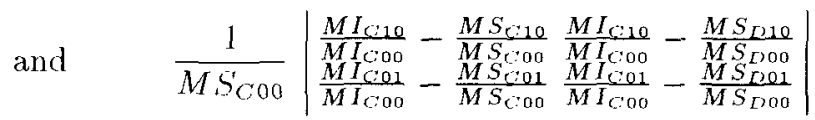

\section{Photometric Invariants.}

Photometric scaling : $M I_{10} / M I_{00}, M I_{01} / M I_{00}, M S_{10}, M S_{01}, M S_{00}$

Photometric scaling + offset $: M S_{10}, M S_{01}, M S_{00}$, and $\frac{M I_{10} M S_{00}-M I_{00} M S_{10}}{M I_{01} M S_{00}-M I_{00} M S_{01}}$

\section{Affine / Photometric Invariants.}

Photometric scaling:

3 shape moments: $M S_{C 00}^{\prime} / M S_{D 00}$, and $M S_{C 00} / M S_{E 00}$

1 intensity +2 shape moments : $M S_{C 00} / M S_{D 00}$, and (3)

2 intensity +1 shape moments:

$$
M I_{C 00} / M I_{D 00} \quad \text { and } \quad \frac{1}{M S_{C 00}}\left|\begin{array}{l}
\frac{M I_{C 10}}{M I_{C 00}}-\frac{M S_{C 10}}{M S_{C 00}} \frac{M I_{C 10}}{M I_{C 00}}-\frac{M I_{[D 10}}{M I_{D 00}} \\
\frac{M I_{C 00}}{M S_{C 00}} \frac{M I_{C 01}}{M I_{C 00}}-\frac{M I_{L 01}}{M I_{[100}}
\end{array}\right|
$$

3 intensity moments: $M I_{C 00} / M I_{D 00}$, and $M I_{C 00} / M I_{E 00}$

2 intensity +2 shape moments : $M I_{C 00} / M I_{D 00}, M S_{C 00} / M S_{D 00},(3)$

(3) with the rôles of $C$ and $D$ reversed, and

$$
\left|\begin{array}{l}
\frac{M I_{C 10}}{M I_{C 00}}-\frac{M S_{C 10}}{M S_{C 00}} \frac{M I_{D 10}}{M I_{D 00}}-\frac{M S_{C 10}}{M S_{C 00}} \\
\frac{M I_{C 01}}{M I_{C 00}}-\frac{M S_{C 01}}{M S_{C 00}} \frac{M I_{D 01}}{M I_{D 00}}-\frac{M S_{C 01}}{M S_{C 00}}
\end{array}\right|:\left|\begin{array}{l}
\frac{M I_{C 10}}{M I_{C 00}}-\frac{M S_{C 10}}{M S_{C 00}} \frac{M S_{D 10}}{M S_{D 00}}-\frac{M S_{C 10}}{M S_{C 00}} \\
\frac{M I_{C 00}}{M I_{C 01}}-\frac{M S_{D 01}}{M S_{C 00}}-\frac{M S_{C 01}}{M S_{D 00}}-\frac{M S_{C 00}}{M C}
\end{array}\right|
$$

Photometric scaling + offset $: M S_{C 00} / M S_{D 00}$,

$$
\frac{M I_{C 00}}{\left(M I_{C 00} M S_{D 00}-M I_{D 00} M S_{C 00}\right)}\left|\begin{array}{l}
\frac{M I_{C 10}}{M I_{C 00}}-\frac{M S_{C 10}}{M S_{C 00}} \frac{M S_{C 10}}{M S_{C 00}}-\frac{M S_{D 10}}{M S_{D 00}} \\
\frac{M I_{C 01}}{M I_{C 00}}-\frac{M S_{C 01}}{M S_{C 00}} \frac{M S_{C 01}}{M S_{C 00}}-\frac{M S_{D 01}}{M S_{D 00}}
\end{array}\right|
$$

(4) with the rôles of $C$ and $D$ reversed, and

$$
\frac{M I_{C 00} M I_{D 00} M S_{D 00}}{\left(M I_{C 00} M S_{D 00}-M I_{D 00} M S_{C 00}\right)^{2}}\left|\begin{array}{l}
\frac{M I_{C 10}}{M I_{C 00}}-\frac{M S_{C 10}}{M S_{C 00}} \frac{M I_{D 10}}{M I_{D 00}}-\frac{M S_{D 10}}{M S_{D 00}} \\
\frac{M I_{C 01}}{M I_{C 00}}-\frac{M S_{C 01}}{M S_{C O 0}} \frac{M I_{D 01}}{M I_{D 00}}-\frac{M S_{D 01}}{M S_{D 00}}
\end{array}\right|
$$




\subsection{Second-order Moment Invariants}

\section{Affine Invariants :}

2nd order moments of 1 type only :

$$
\frac{\left(M_{20} M_{00}-M_{10}^{2}\right)\left(M_{02} M_{00}-M_{01}^{2}\right)-\left(M_{11} M_{00}-M_{10} M_{01}\right)^{2}}{M_{00}^{6}}
$$

2nd order moments +0 th order moment of other type : $M I_{00} / M S_{00}$, and (5) 2nd order intensity moments +1 th order shape moments:

$M I_{00} / M S_{00},(5)$ for intensity moments, and

$$
\frac{\beta \epsilon^{2}-2 \alpha \delta \epsilon+\gamma \delta^{2}}{M S_{00}^{8}}
$$

where

$$
\begin{array}{lll}
\alpha=\left(M I_{11} M I_{00}-M I_{10} M I_{01}\right), & \delta=\left(M S_{00} M I_{10}-M S_{10} M I_{00}\right), \\
\beta=\left(M I_{20} M I_{00}-M I_{10}^{2}\right), & \epsilon=\left(M S_{00} M I_{01}-M S_{01} M I_{00}\right), \\
\gamma=\left(M I_{02} M I_{00}-M I_{01}^{2}\right) . & &
\end{array}
$$

2nd order intensity moments + 2nd order shape moments :

$M I_{00} / M S_{00}$, (5) for intensity moments, (5) for shape moments,

(6), (6) with the rôles of intensity and shape moments reversed, and

$$
\frac{(\alpha A-\gamma B)^{2}+\left(\alpha A-\beta C^{\prime}\right)^{2}+2(\beta A-\alpha B)\left(\gamma A-\alpha C^{\prime}\right)}{M I_{00}^{6} M S_{00}^{6}}
$$

where

$$
\begin{gathered}
A=\left(M S_{11} M S_{00}-M S_{10} M S_{01}\right), B=\left(M S_{20} M S_{00}-M S_{10}^{2}\right) \\
\text { and } \quad C^{\prime}=\left(M S_{02} M S_{00}-M S_{01}^{2}\right) .
\end{gathered}
$$

\section{Photometric Invariants.}

Photometric scaling : $M I_{10} / M_{00}, M I_{01} / M_{00}, M I_{20} / M_{00}, M I_{11} / M_{00}, M I_{02} / M_{00}$ Photometric scaling + offset : $P I(00,10,01), P I(00,10,20), P I(00,10,11)$, $P I(00,10,02), P I(10,01,20), P I(10,01,11), P I(10,01,02), P I(01,20,11)$, $P I(01,20,02)$, and $P I(20,11,02)$, where

$$
P I(i j, k l, m n)=\left|\begin{array}{ll}
M_{I i j} & M_{I k l} \\
M_{S i j} & M_{S k l}
\end{array}\right|:\left|\begin{array}{ll}
M_{I i j} & M_{I m n} \\
M_{S i j} & M_{S m n}
\end{array}\right|
$$

\section{Affine / Photometric Invariants.}

Photometric scaling:

2nd order intensity moments +0 th order shape moment :

$$
\frac{\left(M I_{20} M I_{00}-M I_{10}^{2}\right)\left(M I_{02} M I_{00}-M I_{01}^{2}\right)-\left(M I_{11} M I_{00}-M I_{10} M I_{01}\right)^{2}}{M I_{00}^{4} M S_{00}^{2}}
$$


2nd order intensity moments +1 th order shape moment: $(8)$, and

$$
\frac{\beta \epsilon^{2}-2 \alpha \delta \epsilon+\gamma \delta^{2}}{M I_{00}^{4} M S_{00}^{2}}
$$

with the same notations as in (6).

2nd order intensity moments + 2nd order shape moment : (8), (9),

(5) for shape moments, (9) with the roles of intensity and shape moments reversed, and

$$
\frac{(\alpha A-\gamma B)^{2}+(\alpha A-\beta C)^{2}+2(\beta A-\alpha B)(\gamma A-\alpha C)}{M I_{00}^{4} M S_{00}^{8}}
$$

with the same notations as in (7).

Photometric scaling + offset : the simplest (of the 4 existing) invariant(s) is

$$
\frac{M S_{00}^{4}\left(\bar{\beta} \bar{\epsilon}^{2}-2 \bar{\alpha} \bar{\delta} \bar{\epsilon}+\bar{\gamma} \bar{\delta}^{2}\right)}{(\bar{\alpha} A-\bar{\gamma} B)^{2}+(\bar{\alpha} A-\bar{\beta} C)^{2}+2(\bar{\beta} A-\bar{\alpha} B)(\bar{\gamma} A-\bar{\alpha} C)}
$$

with $A, B, C$ as defined earlier for eq. (7) and

$$
\begin{aligned}
& \bar{\alpha}=\left(M I_{10} M S_{00}-M S_{10} M I_{00}\right)\left(M I_{01} M S_{00}-M S_{01} M I_{00}\right), \\
& \bar{\beta}=\left(M I_{10} M S_{00}-M S_{10} M I_{00}\right)^{2} \quad \bar{\delta}=M S_{00}\left(M I_{10} M S_{00}-M S_{10} M I_{00}\right) \\
& \bar{\gamma}=\left(M I_{01} M S_{00}-M S_{01} M I_{00}\right)^{2} \quad \widetilde{\epsilon}=M S_{00}\left(M I_{01} M S_{00}-M S_{01} M I_{00}\right)
\end{aligned}
$$

\section{Experiments}

Due to space restrictions, only one experiment will be discussed here. Other experiments are given in [13]. The goal of this experiment is to test the discriminatory power of invariant (4). To this end, its value is computed for images of postcards. Fig. 2 shows four of the postcards used in the test. To test the invariance of the expression, each of the images is transformed mathematically for several combinations of affine transfomations as well as photometric scaling and offset. The original image and three of its transformed versions are shown in Fig. 3. The intensity pattern of the postcard is delineated by an parallellogram fitting program. In the experiment, the invariant (4) is calculated for the original image and its transformed versions. Since this invariant needs two contours $C$ and $D$, the postcards's outline is taken as one contour and a parallellogram corresponding to one half of the postcards is used for the second contour. As there are 4 possible choices for this second part, which cannot be distinguished in an affine context. Therefore, (4) is computed for each of the four possibilities and their values are summed. The results of this computation are given in Table 1 for the four postcards shown in Fig. 2 and their corresponding transformed versions as the one shown in Fig. 3. Clearly, the values of this symmetric invariant expression are quite stable over the different transformations, whereas the value is significantly different for the different postcards. 

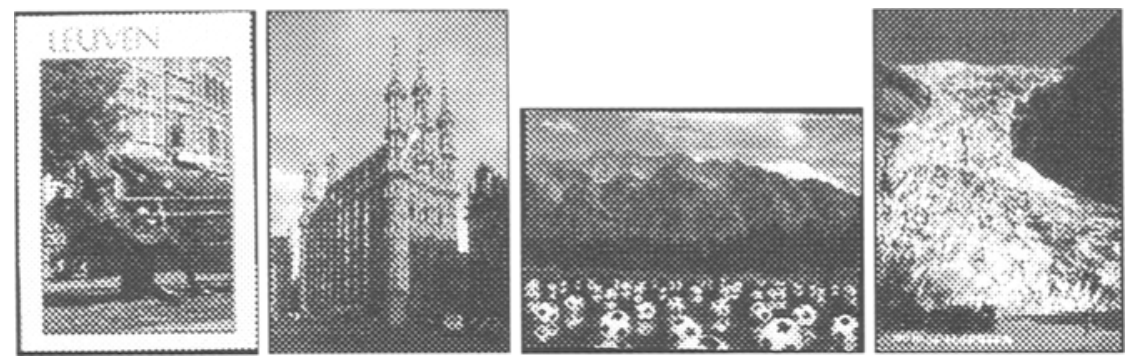

Fig. 2. Four postcards used in the test.
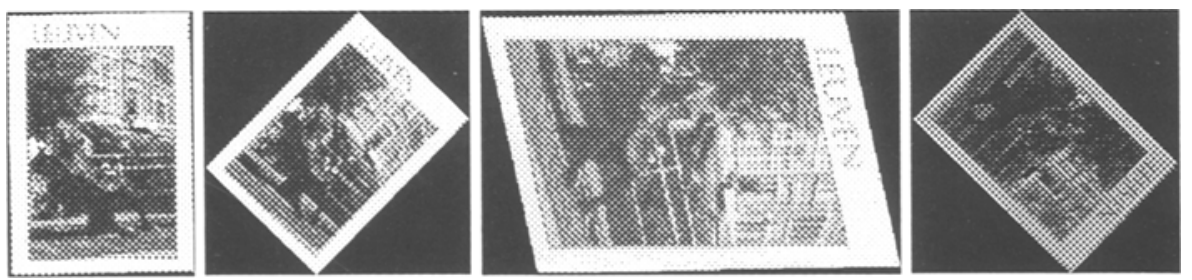

Fig. 3. The original and three transformed versions of the first postcard in Fig. 2.

\section{Conclusions}

The central theme of the paper is the generation and classification of simple and robust moment invariants by (1) the deliberate mixing of intensity and shape moments, and (2) the provision for joint invariance under geometric (affine) deformations and photometric changes,

The mixing of intensity and shape moments has a number of advantages. First, it is a necessary condition for the use of intensity moments in combination with offsets in the photometric changes, a fact that does not seem to have been made explicit in the literature. Without the shape moments no group action would result. Second, mixing both moment types as a deliberate strategy leads to their optimal use and simpler moment invariants, whereas their occasional mixing in the past seems to have been rather ad hoc and thereby not exploited to the full.

Table 1. Values of the invariant (4) for the postcards in Fig. 2 and their transformed versions as in Fig. 3.

\begin{tabular}{|l|rccc|}
\hline & original & transform 1 & transform 2 & transform 3 \\
\hline postcard 1 & 0.224 & 0.230 & 0.225 & 0.210 \\
\hline postcard 2 & -1.818 & -1.814 & -1.850 & -1.840 \\
\hline postcard 3 & 0.590 & 0.587 & 0.592 & 0.560 \\
\hline postcard 4 & 0.125 & 0.130 & 0.121 & 0.133 \\
\hline
\end{tabular}


It has been observed that the more discriminating moment invariants may be of higher order than the second. Thus, there is a conflict between robustness against noise and discriminant power. As the experiments have shown, the lower order invariants proposed here are effective, especially since there are more of them available now.

\section{References}

1. A. Abo-Zaid, O. Hinton, and E. Horne, About moment normalisation and complex moment descriptors, Proc. 4th International Conference on Pattern Recognition, pp.399-407, 1988.

2. J. Flusser and T. Suk, Pattern recognition by affine moment invariants, Pattern Recognition, Vol. 26, No. 1, pp.167-174, 1993.

3. M. Hu, Visual pattern recognition by moment invariants, IRE Trans. Information Theory, IT-8, pp.179-187, 1962.

4. S. Maitra, Moment invariants, Proc. IEEE, Vol.67, pp.697-699, 1979.

5. J.L. Mundy and A. Zisserman (eds.), Geometric Invariance in Computer Vision, MIT Press, Cambridge, Massachusetts, 1992.

6. J.L. Mundy, A. Zisserman, and D. Forsyth (eds.), Applications of Invariance in Computer Vision, Lecture Notes in Computer Science, Vol. 825, pp. 89-106, Springer-Verlag, Berlin / Heidelberg / New York / Tokyo, 1994.

7. M. Oren and S. Nayar, Seeing beyond Lambert's law, Proc. European Conference on Computer Vision, pp. 269-280, 1994.

8. R. Prokop and A. Reeves, A survey of moment-based techniques for unoccluded object representation and recognition, CVGIP: Models and Image Processing, Vol.54, No.5, pp. 438-460, 1992.

9. T. Reiss, Recognizing planar objects using invariant image features, Lecture Notes in Computer Science, Vol. 676, pp. 89-106, Springer-Verlag, Berlin / Heidelberg / New York / Tokyo, 1993.

10. H. Schulz-Mirbach, Anwendung von Invarianzprinzipien zur Merkmalgewinnung in der Mustererkennung, VDI Verlag, 1995.

11. C.-H. Teh and R. Chin, On image analysis by the methods of moments, IEEE Transactions on Pattern Analysis and Machine Intelligence, Vol. 10, No. 4, pp. $496-513,1988$.

12. L. Van Gool, T. Moons, E. Pauwels and A. Oosterlinck, Vision and Lie's approach to invariance, Image and Vision Computing, Vol. 13 (1995), no. 4, pp. 259-277.

13. L. Van Gool, T. Moons, and D. Ungureanu, Affine/photometric invariants for planar intensity patterns, K.U.Leuven Technical Report KUL/ESAT/MI2/9511, Katholieke Universiteit Lenven, Belgium, 1995.

14. L. Wolff, On the relative brightness of specular and diffuse reflection, Proc. Computer Vision and Pattern Recognition, pp.369-376, 1994. 